\title{
Neologismen und Modewöster in der deutschen Sprache
}

\section{Reinaldo Bossmann}

Sprache ist Manifestation unseres Denkens. Sie dient der Kommunikation. A!s Instrument der Ausserung unse:er Gedanken- und Gefühlswelt ist sie ständig Veränderungen unterworfen. Sie muss mit der Kreativität des menschlichen Geistes, ganz gleich ob dieser sich unter positiven oder negativen Vorzeichen vollzieht, Schritt halten.

Die folgenden Beispiele für Neologismen und Modewörter sind grosse Ausdehnung des sprachlichen Niederschlags. Er erfasst alle Schichten der Gesellschaft. Mit der propagierten "Ware" werden Begriff und Name des "Produkts" geliefert. Sprachliche Neuschöpfungen dringen in die Umgangssprache ein, behaupten sich oder werden bald vergessen, je nach Trend, Tendenz und Wichtigkeit der Thematik.

Die folgenden Beispiele für Neologismen und Modewörter sind meist Auszüge aus den Informationsbläitern DER SPIEGEL, DIE ZEIT und UNSERE ZEITUNG, sie stammen aus den Jahren 1974 bis 1977.

Die zu diesem Thema erschienene Literatur, insbesondere die Arbeiten von Bełz, Benckiser, Carstensen, Galinski, Stave, Weigel, Wills und Wellmann, waren mir in Brasilien nicht zugänglich; auch Nachfragen in Deutschland ergaben, dass diese bereits vergriffen seien. Den Kollegen Prof. Dr. Stötzel, Universitäł Düsseldorf, Dr. Stickel, Institut fur Deutsche Sprache, Mannheim, und Dr. Latzel, Goethe-Institut, München, danke ich auf diesem Wege bestens für einige Auskünfte.

Die Suche nach "echten" Wortneuschöpfungen war nichi sehr crgiebig. Was vor!iegt, ist meist dem Englischen und Jiddischen entnommen, offenbart wenig Phantasie, vielfach sind es schon altbekannte, wieder mit anderem Bedeutungsinhalt in die deutsche Sprache eingeführte Wörter. Einige sind typische SPIEGEL-Wörter, andere aus der Szhicht des Jargons mit deutlichem Berufs- und Szenenhinweis. 
Reichlicher war die Ernte an Modewörtern, die sich entweder zäh als "Spitze" behaupten oder, was in de: Natur ihrer Kennzeichnung liegt, allmählich abklingen, durch neve ersetzt werden, wie es Zeit- und Gesellschafisströmungen und Auswirkungen von besonderen Ereignissen erfordern.

\section{Neologismen:}

Auf einem Bahnsteig der U-Bahn im Münchener Zentrum steht auf einem Schild Nothalt, um einen Zug im Bedarfsfall zum Stehen zu bringen. Das Wort erinnert an Notbremse, Notverordnung, Notstand, Notwehr usw. In diesem Fall hat sich die Direktion der dortigen Verkehrsbetriebe etwas einfallen lassen.

Jeuen für spielen isł eine wenig g'ückliche Neubildung; eigenartig ist, dass dieses Wort nicht vom französischen Verbum jover spielen, sondern vom Substantiv jeu - Spiel gebildeł wurde.

Das Verb orgasmen gehört zu Orgasmus und ist ein häufig gebrauchtes Wo:t in der Sex-Szene: Um zu orgasmen, solle sich die Frau in jedem Falle selbst behelfen und aktiver werden als bisher. ${ }^{1}$ )

Ertrügen, wie man in München sagt, wird im Jargon der Untersuchungsbehörden neben betrügen gebraucht: Wir haben es mit einem Star zu tun, mit einem Rekordaufsteller, mit einem Mann, dem es gelungen ist, mindestens zwölf Millionen Mark zu erirügen. ${ }^{2}$ )

Der Jargon der Rauschgift-Szene kennt dealen neben englischem deal und dealer: Der Kriminalist vom Rauschgiftdezernat kennt die Route, auf der Heroin ins Land kommt, er weiss, wo Dealer dealen und kann unter Umständen als Käaufer auftreten. ${ }^{3}$ ) Für Dealer gibt es die Kategorien: Kleindealer und Grossdealer.

Zu englisch clean wurde cleanen gebildet und stammt aus dem Terroristen-Jargon: Lager 1 war Ende November, wie die Papiere besagen, aufgegsben worden (Haag-O:der: Auflösen, cleanen, ordrungsgemäss abmelden. $\left.{ }^{4}\right)$

Erich Kästner hat schimpfen zu schimpfieren umgewandelt, die Apos haben die Sprache um schubladieren bereichert: Wenn sich die Berliner Studenten aus dem fatalen Zwang zur Affirmation als W:derspruch lösen wollen, dann düifen sie sich nicht auf positive $\mathrm{Ge}$ -

1) Aus: Hite-Report, in: DER SPIEGEL, Nr. 37, v. 5-9-1977.

2) Aus: Wir wollen nicht streiten. In: DER SPIERGEL, Nr. 9, v. 21-9-1977.

3) Aus: Eigentlich müsste jeder verdaechtig sein. In: DER SPIEGEL, Nr. 38, v. 12.7-1977.

4) Ebenda, siehe Fussnote 3. 
genbilder berufen und sich schubladieren lassen als Radikalinskis, Schwachköpfe und Pervertierte. ${ }^{5}$ )

Mit begrasmarmein ${ }^{6}$ ) $=$ sich begraben lassen wurde ein altes deutsches Wort durch Bundesfinanzminister Hans Apel wieder zu neuem Leben erweckt. Oberhaupt zeigt die heutige Umgangsspiache die Tendenz, Verben mit dem Präfix be-, dem soganannten inhumanen Akkusativ, zu verwenden. In Firmeninseraten liest man: bestuhlen, bedachen, bedecken, bebauen u.a.

Viele SPIEGEL-Wörter ${ }^{7}$ ) wie abblocken für verhindern und das englische Flop für Hinplumpsen (im SPIEGEL für Versager) sind auch in die neuesten Ausgaben des Duden-Wörterbuches aufgenommen worden.

Mutterneutral ist eine Worlschöpfung des Finanzgerichts in Berlin vom 3. Juni 1976, Az I 345/75: Das mutteineutrale Ehegatten-Splitting genügt nicht. Eine saftige Jungfernkindersteuer muss an die Stelle des Kindergeldes treten, um dieses ha:tnäckige Emanzenpack zum Heira:en zu zwingen. ${ }^{8}$ )

Zu Kassiber wurde kassibern (jidd., Gaunersprache) gebildet: Ihretwegen, weil etliche in Verdacht gerieten, für einsitzende Terroristen zu kassibern, sann der Gesetzgeber auf rigorose Einschränkung angestammter Ve:teidigerrecht. ${ }^{\circ}$ )

An SPIEGEL-Neuwörtein sind zu erwähnen: Big Raushole (dt.engl. Mischung), Kob (Abkürzung für Kontaktbereichsbeamter). Tätereinstrom (Kripo-Jargon), Freiballöner (Sport-Szene), Pharmakadaver

5) Aus: Schwat:er, Schaudenker, Generalist (SPIEGEL, Nr. 37, v. 5-9-77).

6) SPIEGEL, Nr. 6. S. 3.

7) SPIEGEL, Nr. 6, 1977, S. 3.

8) DER SPIEGEL, Nr. 40, 1977.

9) In: Mord beim boesen Wort (SPIGEL, Nr. 6, 1977, S. 28. 
(Medizin, Pharmazie), Prägeriatrie (Medizin), Engelskreis Wirtschaft) als Gegensatz zum Teufelskreis und breaken (Rundfunk als Trend zum Eigensender, Volksliebhaberei).

Verkarten (Spionage_Jargon), vermarkien (Wirtschaftsszene), beide im Duden-Wörterbuch 1973 bereits registriert, sind im Hamburger Informationsblatt DIE ZEIT häufig verwendete Verben, während missmanagen und mitmakeln oft im SPIEGEL anzutreffen sind. Makeln und managen sind auch im Duden 1973 ve:zeichnet, ebenso schönen, das in diesem Nachrichtenmagazin als geschömte Gruppenversicheiungen und mosern als anmosern, bosseln als herumbosseln vorkommen.

Müllem will nicht heisen, Gymnastik nach den Vorchriften des dänischen Gymnastiklehrers J.P. Müller zu treiben, sondern will besagen, solange Gerd Müller Tore schiesst, müllert er. ${ }^{10}$ )

Aus dem Englischen kommen flaaten (floatende Wechselkurse, Aufivärtsfioa:en der DM, des SFr.), relaxen, talken und Storys.

Cankie: srscheint neben umgangssprachlichem Banker, Residenz neben Residentur (Terrorisienszene). Mord neben Morderei, von Knast w/urde Knastologen gebildet, überhaupt Knast ist "in", wie DIE ZEIT schreibt, und die Verben vorformulieren, konditionieren und umfunktionieren, gravieren (gravierender Vorgang), observieren (Staałsschütze:-!argon), onias:en (angelastete Wirtschaftsschwierigkeiten), eskaladieren, tabuisieren, enttabuisieren, frustrieren, verunsich or n unser Leben, sind aber Punk, das Letzie vom Letzten (Punk ist der Varsuch, allem die Krone aufzusetzten). Konditionieren heisst, jemanden so drillen, dass er sich einer gewissen Aufgabe refiexhaft unterzieht. Vermutlich wurde das Wort in diesem Sinne aus ciem Amerikanischen übernommen. Ein Kindergarten oder eine Organisation werden unfunktionient, d.h. eine Sache oder Einrichtung in einer ande:en als c'e: ursprünglich vorgesehenen Funktion zu verwenden: eine Vorlesuric umfunktionieren; einen Professor zu einem Genossen umfunkticnieren: ihm eine andere Wel:anschauung zu geben. Ist er umfunktionictt, dann ist er eigentlich nicht mehr Professor. Aus

10) In: Der Bomber der Nation. DiE ZEIT, vom 6. Dez. 1974. 
der Sicht einiger "Studenten" ist die Aufgabe eines Professors, die Studenten zu reprimieren.

\section{Altbekannte Wörter z.T. mir anderem Bedeutungsinhalt:}

Festmachen hat den Sinn von vereinbaren; neuerdings ist es aber - mit gewissen Einschränkungen - identisch mit anbinden. Es stammt aus der "Frankfurter Soziologischen Schule". Studium erscheint in den Zusammensetzungen Kontaktstudium, Infensivstudium und Streustudium. Kontäktstudium bezieht sich nicht auf Studenten, sondern is! für Akademiker bestimmt, die wieder zur Universität kommen, um sich über ihr Fach zu informieren und ihr Fachwissen aufzufrischen. Für Kurse von kurzer Dauer, meistens war die Ferienzeit dafür vorgesehen, hat man jahrelang in Brasilien reciclagem - atualização pedagógica - veiwendet, bis sich dieses Wort zum Uberdruss abgenutzt hat. Das Kontaktstudium kann durchgeführt werden: als In!ensivstudium (Intensivkursus) und als Streustudium, das immer eine längere Zeit voraussetzt. Projekt ist PIanung, Entwurf, Vorhaben, unter Studenten (Universität Düsseldo:f) die Applikation eines Planes. Man spricht vom Projektleiter, von Projektenmachern und von der Projektgruppe. Kommune bedeutet eine gemeinschaftliche Studentenwohnung, auch für Studentinnen, mit Sex- und auch ohne Sexbetrieb, in den 60er Jahien sehr modisch, heute stark im Abklingen, und man zieht Wohngemeinschaft vor. In Brasilien heisst die typische Studentenwohnung república, allerdings noch nach Geschlechtern getrennt und ohne Sexbetrieb. Umwell, im Land unter dem Kieuz des Südens meio ambiente, wird gleichgesetzt mit Umweltver. schmutzung Umweltvergifyung: Hier stinkt's, das ist wieder die Umwelt! Und als Zusammenseizungen erscheinen: Umweltbewusstsein, Umweltschutz, umweltbedingt, Umwelteinfluss, umweltneutral, umweltschädlich u.a. Lebensqualität, aus dem Politiker-Jargon, bezieht sich nicht nur auf die Gesundheit und das Materielle, sondern auch darüber hinaus auf den Arbeitsplatz, diesen z.B. humaner zu gestalten. Bert Brecht wurde als Stückeschreiber weltbekannt. Die Ubertra. gung des Handwerklichen auf das Geistige setzt sich immer mehr durch: Textemacher, ein ge!ernter Philosoph, ein ge!ernter Studienrat, machbar, das Machbare, der Macher, und "er macht in Obstruktion".

Hierzu gehört auch: Sinn machen für Sinn haben: Zum Belgrader Nachfolge-Treffen der Konferenz über Sicherheit und Zusammenarbeit in Europa (KSZE) meinten beide (Gensche: und Gromyko) übereinstimmend, es mache keinen Sinn, sich gegenseitig anzuklagen. 
Die Touristik, die für manche Länder Devisenbringer Nr. 1 ist, wird auch die weisse Industrie genannt, und "es ist schon fast so weit, dass das Wort Tourist nicht nur hierzulande ein Schimpfwort geworden ist". ${ }^{11}$ )

In diesem Zusammenhang spricht man auch von geldigen Frem. den mit ihren hingeklotzten Zweitwohnsitzen, die die Region durch Verramschung ${ }^{12}$ ) (Naturschützer_Jargon) bedrohen. Die Gekünstelte Lederhosen-Architektur ${ }^{13}$ ) verdrängt zusehends die bäuerlichbarocke Baukunst vergangener Zeiten. Planer fürchten, dass die betonierten Bereiche zu einer einzigen multinationalen Alpinopolis zusammenwachsen. ${ }^{14}$ ) Und was sind Jodlerhäuser (Architekten-Jargon)? 'Wer echtes Almvieh sehen will, muss oft erst einen kilometerbreiten Ring corffremder Randsiedlungen durchwandern, in dem die rustikal maskierten konfektionierten Jodlerhäuser vorherrschen. ${ }^{13}$ )

Zu austauschen, Austausch wurde Freitausch, zu Erich von Däniken die Dänikitis, zu Idiologisierung Entidlologisierung und zu Entscheidung die End-Entscheidung geprägt.

Jede Art von Jargon kennt viele Wörter, die auf Anhieb sinnge. mäss nicht $z U$ verstehen sind, da sie sich semantisch verändert haben. Uber einige berichtete DIE ZEIT' ${ }^{19}$ ) Aus dem Männerjargon: angebohrter Zahn, angebuffte Tomate für ein "e:obertes" Mädchen und Zapfenstreich für Vergewaltigung. Aus dem "Wörterbuch für Knackis - Zeitliches aus Hannover" (Artikel von Joachim Holtz im Hamburger Informationsblatt DIE ZEIT) sind zU nennen: Ambach ist das "Wissen was los ist"; Schliessfach ist Zelle; viel Qualm vor der Brust haben bedeutet eine lange Haftstrafe vor sich haben; der Aze ist ein Freund mit gleichem Schicksal und ein Klimbimski gehört zu den Kleinen aus der Unterwelt. Babalu ist der Regengott, zu dem die Knackis flehen, damit sie nicht im Freien arbeiten müssen, und figine machen

11) UNSERE ZEITUNG, Nr. 247, August 1977.

12) DER SPIEGEL, Nr. 9, 1977, S. 68.

13) Ebenda, s. Fussnote 12.

14) DER SPIEGEL, Nr. 9, 1977, S. 63.

15) DER SPIEGEL, Nr. 9, 1977, S. 67.

16) vom 5.11.1976, in: Varianten der Notzucht. 
sagen sie für die Vortäuschung eires Defektes, mit dem sie sich zum Dachdecker, zum Psychologen, schicken lassen, und dieser meikt, ob es sich um einen Laumann, um einen undurchsichtigen Menschen oder um eine Knastmauke, eine Haftpsychose, handelt.

\section{Aktuelle Modewörter:}

Seit dem Präsidentenwechsel in den USA und der Geiselnahme Schleyers wurden Menschenrechte und Sympathisanten des Terrors und der Terroristen zu den am meisten gebrauchten Modewörtern durch die Massenmedien und der Bevölkerung. Unter Bundeskanzler Willy Brandt machte die Ostpolitik tägliche Schlagzeilen, während unter dem jetzigen Bundeskanzler Helmut Schmidt, le Feldwebel, wie ihn die französische Presse nennt, das Wort Berufsverbot stark in den Vordergrund des politischen Geschehens in der Bundesrepublik Deutichland trat. Ostpolitik, Berufsverbot, le Hexenjagd (in bezud auf das Berufsverbot) gingen sogar als Lehnwörter ins Französische und Englische ein. In der Tat ist ein beliebter Ausdruck Helmuł Schmidts, während Walter Ulbricht häufig das ist ein Fakt gebrauchte. Die Stammheimer Selbstmorde der Oberterroristen brachten den Suizid in die aufgeheizle Situation und verunsicherten die Bundesrepublik und deutsche Firmen im Ausland. Der Atomvertrag zwischen der Bundesrepublik und Brasilien wurde in der deutschen Presse als Atomgeschäft oder Brasiliengeschäft leider zu häufig als "Geschäft" herausgestellt. In diesem Zusammenhang wurde auch von aufstülpen geredet: Die Deutschen möchten dem unverändeiten Vertrag mit Brasilia ein internationales Zusatzabkommen aufstülpen". ${ }^{17}$ )

Der Wortschatz der Umgangssprache wird sietig durch Fachsprachen und Jargons angereichet. Jeder Wissenschafts- und Berufszweig hat seine eigene Fachsprache, die nur dem Fachmann geläufig ist. Dem Sprecher der Umgangssprache bleiben solche sprachgebundenen Terminologien und termini technici unversłändlich (besonders aus der Pädagogik, Soziologie, Psychologie und Technik). Um diese sich anzueignen und die Verständigungsschwierigkeiten zwischen Umgangssprache und Fachsprachen zu übe:brücken, sind Wörterbücher der verschiedenen Sondersprachen längst ein notwendiges Ubel geworden. Für die Forschung, zur weiteren Entwicklung der Wissenschaft ist die Sondersprache für den Fachmann von essentieller Bedeutung. Dem Laien aber kann kaum zugemutel werden, um die bestehende

17) Der Spiegel, Nr. 6, 1977, S. 29. 
Sprachkluft zu beseitigen, eine Sondersprache unbedingt zu erlernen.

Wörter aus der Umgangssprache, den Fachsprachen und Berufsjargons kommen in Brauch, werden zu Modewörtern, bewahren einige Zeit diesen Status, um später abzuklingen und aus dem Usus zu verschwinden. Alles, was Mode ist - eine Binsenweisheit - wird durch die Publikationsorgane angeheizt. Der Brauch, das Neve zu bevorzugen, lässt das Alte schnell vergessen. Zeitgeist und Tagesgeschehen liefern dazu den nötigen Beitrag. Mit der Gründung der Bundesrepublik Deutschland beherrschten de: Wiederaufbau auf allen Gebieten, die Ankurbelung der Wirtschaft, der Marshall-Plan, die Wiedervereinigung der beiden deutschen Staaten, schliesslich das deutsche Wirtschaftswunder, die verschiedenen Wellen (Fresswelle, Bekleidungswelle, Möbelwelle, Urlaubswelle usw.), vor mehr als einem Jahrzehnt die Mauer Denken und Handeln der Politiker und Bundesrepublikaner. Wer spricht heute noch davon? Ende der 60er Jahre waren es die Studentenunruhen, die Apo-Welle, die die Ruhe in der Bundes:epublik störten. Gegenwärtig (September, Okłober, November 1977) sind es Terror, Geiselnahme, Sympathisanten der Terroristen, Zielfahndung Kontaktsperregesetz, Baustopp für Kernkrafiwerke, Ablagerung von Atommüll, Vorlesungsboykott, Hochschurahmenge. setz, Demonstration (im Sinne von Protest), Konjunktur, Arbeitsiosenquote, Mitbestimmung, Numerus clausus und Uberkapazitäten, die als Modewörter, auch mit ihren zahlreichen Komposita, in Erscheinung treten. Andere Geschehnisse, andere Probleme, die einer vorrangigen Lösung bedü:fen, werden wieder andere Wörter an die Oberfläche spülen, so dass der Kreislauf des Kommens und Gehens, des Auf-und Abtauchens im sprachlichen Wechselspiel erhalten bleibt.

In Zeitungen und Zeitschriften besonders oft angetroffene Wörter in den Jahren von 1974 bis 1977 waren:

Aus der Politik: Ordoliberal, taktieren, Offerte, Geisterschreiber (wörtliche Ubersetzung von ghost-writer), Revirement, Negativtrend, spitzenjob, Zweckbündnis, Meriten (port. méritos), multilateral, frustrieren, Frustration (in Brasilien auch Modewörter: frustrar, frustração), Freiheif und Sozialismus. (Thema als Wahlpropaganda), Grundsatzerklärung und Eurokommunismus.

Aus der Wirtschaft: Kosakenrubel für Ostmark (Spiegel-Wort), Bau-Mafia, Händler-Mafia, Schleichwerbung, Konjunkłur, Stabilität, revozieren für wideriufen, vermarkten, verkraften, florieren, Block- 
Verbund, Siruktur (-Korsett), Abschlag, Desaster, Talfahrt, Wohnungshalden, Kontingent, Graumarkt, Kostenanstieg, Multis (multinacionais in Brasilien), Establishment, Recycling, Shopping-Touristen, anlasten, manipulie:en, malochen (jidd. für schuften, Spiegel-Wort), Kapitalstrom, Manövriermasse, Pluralismus, arrivieren, akzeptieren, Spots (Fernsehspots), Gerangel, Stringenz, Intransigenz, Ineffizienz, Job und Arbeitslosigkeit.

Aus dem Bildungswesen: Stress (auf allen Gebieten und in jeder Hinsicht), Curriculum, Curricularforschung, Numerus clausus, Studienplatz, Vita (für Lebenslauf, Werdegang), Terror (Leistungsterror, Zensurterror), Mengenlehre, Image (auch Modewort in Brasilien), überfordern, Kontext (contexto in den vergangenen Jahren auch in Mode in Brasilien), Axiom, Assoziativität, Kommutativität, Podiumsdiskussion, Mitbestimmung, Bidungsofferte, Amterkumulation und Hecklastigkeit (in bezug auf die Schulmeister-Schwemme).

Aus der Medizin, Pharmazie und Chemie: Kostenanstieg, exorbitant, Mark-Gigant, Chemie-Multis, Billig-Medizin, Versorgung, Urbanisationsulkus, Schönheitsfehler Kunstgriff, Dämpfung der Kosten, Generica, Geriatrie und Altersversorgung.

Aus der Alkohol - und Rauschgiftszene: Fuselfolklore (SpiegelWort), Pharmaka, Einstiegsdroge, clean, Drogenmeeting, Fixer, Schnupfer, Stoff, Release, Cocaini (Polizei-Jargon für Kokain-Händler), Coqueros (Polizei-Jargon für Kokainsüchtige), Entziehung, Dumpingpreise und Rückfallquote).

Aus der Pornoszene: Voyeur, Fellatio, Edelporno, Lesben (Traditionslesben, Bewegungslesben, Politlesben), Sex, Sex-Symbol und bumsen.

Aus dem Jargon der Staatsschützer, Polizei und des Terrorismus: Desperados, Zellenzirku!are, Infos (Flugblätte:), klammheimlich, Mescalero, Anarchos, Spontis, Raster, Umfeld, Chaoten, Insider, Ambiente (kommt aus dem Künstlerjargon; Im Por'ugiesischen Brasiliens wird ambiente erk!ärt mit: alles, was Lebewesen und Sachen umgibt oder einbezieht; um bom ambiente bezieht sich auch auf die Gesellschaft, in der eine gute Stimmung und Gemü:lichkeit anzutreffen sind), Observation, Zielperson, Treff, Beobachtung, Beobachter, Newcomer, sistieren, han'ierbar, Output (Daten, die eine elekt:onische Datenverarbeitungsmaschine liefert), Combat-Schützen, Lauschtechnik, Wanze 
(installiertes Mikrophon zum Abhören), Vita, konspirativ, Agent, Fahndung, Zielfahndung, Befa (Beobachtende Fahndung), observieren, Construction (Polizei-Jargon für das Anzapfen von Telephonleitungen), Ballermann, Hijacking, Hijacker, Perspektiv-Agenten, Penetrierer (Eindringlinge in gehobene Positionen), R-Agenten (Reisende vor allem für militärische Aufklärung) und Geisel, Geiselnahme, Geiselbefrejung. Geisel hat sinzn Jedeutungswandel durchgemacht: Vornehme, junge Leute, meist aus dem Adel, wurden unter Verbündeten wie unter Gegnern ausge! auscht, wo sie am Hof des Verbündeten oder eines möglichen Gegners aufwuchsen, "um die beiden Staaten miteinander zu verklammern, den Frieden zu sichern, die Einhaltung eines Bündnisses zu garantieren. Sie waren nicht reine Objekte wie die armen Opfer heutiger Banküberfälle oder Flugzeugentführungen, sondern wurden in der Regel so gut gehalien wie die Prinzen oder Höflinge des eigenen Lagers. Es war erwünscht, dass sie sich Sprache, Kulfur und Gewohnheiten des anderen Landes soweit aneigneten, dass sie ciereinst als Verbindungsleute dienen konnten, als Unterhändler oder Gesandte und damit abermals als Boten des Friedens, als Mittler einer erwünschten Verständigung" (Schreiber, Hermann: Die Hunnen Attila probt den Weltuntergang. Econ Verlag, Wien, Düsseldorf, 1976, S. 141/42).

Aus der Sport-Szene: Leistungssport, Image, Trimm-Aktion, Trimmi, trimmen (eigl. aus der Seemannssp:ache), Fitness. Aus der Sprache cier Berline; Motorradfahrer: Auf Strecke (Raten) kaufen, den Hahn aufdrehen $=$ Gas geben, die anderen Gummi riechen lassen $=$ sie ïberholen, absteigen $=$ verunglücken.

Aus dem Englischen: Scrambler (Zerhacker im Funkwesen), Cover-Seiten, Top-New, cronies, Roll-back, Chap (netter Keil), Blow up, Funkfreak, skip names (Tarnnamen), beam, power (als beamen und powern für funken), $C B=$ citiens Band (im Volksmund Blödelband), Hit (Buchhit), Top (Top-Modelle), Callgirl, Dandy, Sit-in, Teachin, high, Cleverness, Flash. Rush-hour, Dressman, huns, teutons, krauts, róds (abfällige Benennungen für die Deu'schen, rads abgeleitet von comrads), Hardware, Get-Rich-Quick-Leute, Welcome-Party und Outlaw (Geächteter, Verfemter).

Altere Modewörter aus den vergangenen Jahrzehnten: Geil ${ }^{18}$ ) war semantischen Veiänderungen im Ablauf der Zeiten unterworfen. Im Mittelalter hatte es die Bedeu:ung von "übermütig, hochgemut,

18) UNSERE ZEITUNG, Nr. 237, vom Oktober 1977. 
üppig", die Neuzeit beschränkt es auf das Geschlechtsleben, den Sex im Sinne von "scharf, brünstig, lüstern"; später wurde es geschlechtlich entkleidet und hat die Geliung von "begierig". In Berlin kommt es in der Zusammensetzung von schwiegergeil vor und bezeichnet eine Muttei, die sich einen Schwiegersohn sehnsüchtig wünscht. Die Soldatensprache kennt es als dienstgeil, Dienstgeilheit für einen gezeigten übertriebenen Diensteifer, während ordensgeil, lamettageil, krempelgeil die Sucht nach Orden und Auszeichnungen und titelgeil die nach mehr Titeln charakterisiert. Zeitungen sind immer schlagzeilengeil, Laufbahnergeizige karrieregeil, Rowdys, vándalos, wie sie in Brasilien genannt werden, sind zers:örungsgeil, und für die Schülergeneration von heute bedeutet ungeil so viel wie "langweilig". Die Negation nicht ungeil ist für eine Peison oder Sache eine Art von Sympathiekundgebung.

Ins Haus stehen ${ }^{19}$ ) für ein bestimmtes Ereignis, das zu erwarten ist, stammt aus dem Jargon der Kartenlegerinnen und Astrologen und ist nach 1947 in die Umgangssprache eingedrungen.

Einen Zahn draufhaben ${ }^{20}$ ) für: sehr schnell fahren, geht auf die Frühzei† der Motorisierung (Kraftfahrerdeutsch) zurück, als der Gashebel, von Hand bedient, an einem gezähnien Kreisausschnitt entlang gefüh:t wurde. Man kann auch einen schönen, guten Zahn draufhaben, je nach Geschwindigkeit. Noch einen Zahn mehr bedeutet das Steigern der Geschwindigkeit und einen Zahn runter das Verringern der Schnelligkeit. In unserem technisierten Zeitalter hört man die Redensa:t auch von Sportlern im Sinne, mehr Kampfkraft zu entwickeln, d. h. einen Zahn zulegen, ferner zur Erhöhung des Einkommens in der privaten und beruflichen Sphäre. Es ist eine Temperamentssache, ob jemand einen Zahn zurückschaltet, oder wer einen Zahn zuviel hat.

Auf den Wecker fallen e:innert an den in den Automaten eingeworfenen Groschen, wobei ein Kingelzeichen er:önt. Wecker vertritt hier "Kopf und Verstand" in den Redensarien: da bleibt der Wecker stehen, der Wecker ist abgestellt, nich: alle auf dem Wecker haben, jemandem auf den Wecker fallen (gehen) und den Wecker anstossen, um neve Lebensenergien zu en:falten.

19) UNSERE ZEITUNG, Nr. 247, August 1977.

20) UNSERE ZEITUNG, Nr. 243, April 1977. 
Die Ende der 60er Jahre aufgekommene Redewendung Du tickst wohl nicht ganz sauber ${ }^{21}$ ) weist ebenfalls auf den Feinmechanismus der Uhr und des Gehirns hin. Damit wird eine Denkfunktion auf das Mechanische übertragen, wenn jemand die Sphäre des Normalen, des Oblichen, sei es durch etwas verrückte Ansichten oder Handlungen, verlässt.

In Zeiten wirtschaftlicher Rezession wird in allen Bereichen immer auf Sparflamme geschallet2), um Mittel (Geld), Stoff (Brennstoff, Treibstoff) und Energie (Kraftanstrengung) einzusparen, was auch für seelische Bereiche wie Begeisterung usw. gilt.

Weg vom Fenster ${ }^{23}$ ), um 1960 aufgekommen, vielleicht im RLhrgebiet, hat vielfache Bedeutung: Alte Leute, vor einigen Jahren nannte man sie Senioren (dazu Senioren- oder Rentnerbetreuung, Seniorenauweis, Senioren-Karneval, Seniorenaktion, Seniorenfahrt u.a.) beobachten nicht mehr die Strasse vom Fenster aus, sie sind krank, vielleicht auch bettlägrig oder sogar schon tot; der Sportler, der Künstler, der weg vom Fenster ist, hat keine Chancen mehr auf seinem Gebiet; für Häftlinge, Schüler, So!daten bedeutet die Redensart, dass sie entlassen sind, für den Spieler, dass er keine Runde auszugeben braucht; für jeden anderen, dass er die Beherrschung verloren, sich "vorbei" benommen hat; für ein Partei mitglied, dass es aus der Partei ausgeschlossen wurde und für einen Angestellten, dass er seine Stelle verloren hat. Wer nicht mehr in der Offentlichkeit erscheint, wer gegen Reformen und Zukunftspläne, auch altmodisch ist, der ist weg vom Fenster.

Mit Senkrechtstarter't) bezeichnet man gewisse Flugzeuge, Hubschrauber, die keine lange Start- und Landepiste brauchen, also fast senkrecht starten und landen, aber auch Politiker, Künstler, im Wirtschaftsleben Tätige, die zu rasch oder kometenhaft emporgekommen sind, Karriere gemacht haben. Die Redewendung gilt auch für eine Ware, einen Artikel, ein Produkt, für eine Fussballmannschaft, die eine starke Erfolgskurve aufzuweisen haben, aber auch für einen

21) UNSERE ZEITUNG, Nr. 226, November 1975.

22) UNSERE ZEITUNG, Nr. 240, Januar 1977.

23) UNSERE ZEITUNG, Nr. 230, März 1976.

24) UNSERE ZEITUNG, Nr. 238, November 1976. 
Menschen, der leicht, ohne Grund, aufbraust.

Als langlebig hat sich Knüller's) erwiesen, das nach dem Ersten Weltkrieg aufkam. Es ist ungefähr mit "Schlager" gleichzusetzen, für etwas Neues mit starker Publikumswirkung, mit besonderer Erwähnung in Presse, Kino und im Verlagswesen; später bekam Knüller die Färbung für etwas Sensationelles, für einen günstigen Kauf zu günstigem Preis, etwa in den Schlussverkäufen. Man bezeichnet auch damit einen spannenden Kriminalroman (in Brasilien romance policial), ein Kriminalstück. Man spricht vom Preisknüller, Knüllerpreis und Knüllerjagd. Nach der Sprachzeitung "Unsere Zeitung" steht das Wort auch oft für ein eindrucksvolles Mädchen in Verbindung mit den Adjektiven "einsam", "satt", "fett" oder "steil" und besagt etwas Unübertreffliches.

Es ist modern, im Zeitalter der Ubertreibung und im Angesicht von gigantischen Leistungen der Technik und Wissenschaft, Ausdrücke im Superlativ zu verwenden, die das Höchste, die "Spitze", echt Spitze, Super-Spitze und sogar irre echte Spitze sind. Der Mensch, im letzten Viertel des 20. Jahrhunderts, lebt mit der Propaganda, der Reklame, und muss diese über sich ergehen lassen. Slogans wie supermodern, superschick, wahnsinnig schick, wahnsinnig billig, Supermarkt, Superstar, Superempfänger, Topmodell sind Stimulantia für Käufe, trotz Arbeitslosigkeit und Talfahrt der Wirtschaft, und demonstrieren die Kaufkraft einer starken Valuta. Die Werbung wird gezielt gerichtet an eventuelle Käuferschichten, durch Umfragen und Mark:unterauchungen ermittelt, nich nur an Teenager, die meist kess, frech, dicklich, aber putzig und prall sind, sondern auch an Alte, an Senioren, die man als Marktpotential entdeckt hat.

\section{R E S U M O}

O artigo trata de Neologismos a Modismos na língua alemä, apresentando exemplos documentados para as duas camadas de palavras em questão.

Este estudo, terminado em 1977, pesquisou o vosabulório alemäo ofertado pelos 3 grandes veiculos de comunicação: DER SP:EGEL, magazine hamburgense de informaç̃o, DIE ZEIT, semanário de Hamburgo, e UNSERE ZEITUNG, jornal lingüístico a informativo da República Federal da Alemanha, Munique. Os meios de comunicarăo, DER SPIEGEL e DIE ZEIT destacam-se pelo uso de um vocabulbrio afual e moderno, quase uniformizado pelas redaçöeș de suas editoras, enquanto que UNSERE ZEITUNG mostra

25) UNSERE ZEITUNG, Dezember 1975. 
fendéncias e correntes - os diversos destinos da palavia slemă, divulgando breves concisos artigos que servem também a aprendizagem do idioma alemão.

Os resultados obtidos evidenciam que neologismos de forma clara bela säo ra:os, conquanto a maioria das palavras em moda pertence à linguagens professionais e à esfera do jargão. Os modismos dependem do decurso de tempo e das disposiçōes políticas, comerciais, culturais e sociais, conforme a importância dos fatos, do valor real do acontecimento e da propaganda feira em forno desses assuntos. 